\title{
Anemia and iron deficiency diagnosis, management and treatment in chronic kidney disease - Consensus and agreement through a Delphi Panel
}

Aníbal Ferreira ${ }^{1}$ on behalf of the group: Ana Farinha ${ }^{2}$, Ana Macedo ${ }^{3,4}$, António Robalo Nunes ${ }^{5}$, José Lopes ${ }^{6}$, Patrícia Branco $^{7}$, Pedro Ponce $^{8}$, Ricardo Neto $^{9}$

${ }^{1}$ Hospital Curry Cabral, Centro Hospitalar Universitário Lisboa Central, Lisboa, Portugal

${ }^{2}$ Hospital da Luz Setúbal, Setúbal, Portugal

${ }^{3}$ Keypoint, Consultoria Científica Lda., Algés, Portugal

${ }^{4}$ Departamento de Ciências Biomédicas e Medicina, Universidade do Algarve, Faro, Portugal

${ }^{5}$ Hospital das Forças Armadas, Lisboa, Portugal

${ }^{6}$ Hospital de Santa Maria, Centro Hospitalar Universitário Lisboa Norte, Lisboa, Portugal

${ }^{7}$ Hospital de Santa Cruz, Centro Hospitalar Lisboa Ocidental, Lisboa, Portugal

${ }^{8}$ NephroCare Portugal, Portugal

${ }^{9}$ Centro Hospitalar Universitário de São João EPE, Porto, Portugal

\section{ABSTRACT}

Background: Anemia is a common complication of Chronic Kidney Disease (CKD), in which iron deficiency's (ID) role is frequently underrated. In CKD, anemia has been associated with higher morbidity and lower quality of life. Nonetheless, reported treatment rates of anemia in CKD are low and guidelines' variability and/or absence for its management and treatment may be preventing patients from receiving optimal treatment. Within this context, we aimed to assess the agreement level on anemia and iron deficiency diagnosis, management, and treatment in CKD patients, by Portuguese physicians in Nephrology, through a Delphi Panel. Methods: A group of seven medical experts in Nephrology and Transfusion Medicine was assembled, and a focus group was conducted, in which 28 statements were agreed upon. Then, a two-round Delphi Panel using a Likert scale was conducted online, inviting Portuguese Society of Nephrology associates to participate. Results: Answers were collected from 76 participants in Round 1 and consensus level was obtained for 1 statement, 57 (75\%) respondents fully disagreeing on transfusing all patients with hemoglobin below $9 \mathrm{~g} / \mathrm{dl}$, regardless of the clinical situation. The remaining 27 statements were used in Round 2, none obtaining consensus level and 14 statements being categorized as qualified majority: 4 on diagnosis, 3 on disease management, and 7 on treatment. Discussion: Our study showed a lack of consensus on diagnosis, management, and treatment of anemia in CKD patients among the nephrology community in Portugal. Overall, our results illustrated the heterogeneity of national clinical practices in: laboratory parameters' choice; cutoff values defining anemia and/or ID; parameter-based therapeutic decisions. Nonetheless, it was shown clear that patient's individual characteristics, clinical settings, and the physician's "clinical sense" seem to be considered to a further extent than the available guidelines. Future studies should be considered to develop recommendations that can be widely accepted.

Keywords: Anemia, Chronic Kidney Diseases, Consensus, Delphi Technique, Iron-Deficiency

\section{INTRODUCTION}

Chronic kidney disease (CKD) is currently defined as decreased kidney function, characterized by a glomerular filtration rate (GFR) lower than $60 \mathrm{~mL} / \mathrm{min} / 1.73 \mathrm{~m}^{2}$ and/or indirect markers of kidney damage for at least three months ${ }^{1,2}$.

Anemia develops as a common complication of CKD. It is defined as hemoglobin levels below the accepted lower level of the normal range ${ }^{3}$, set at $12 \mathrm{~g} / \mathrm{dL}$ in women and $13 \mathrm{~g} / \mathrm{dL}$ in men by the World Health Organization ${ }^{4}$.

Usually anemia prevalence increases, progressively, as kidney impairment worsens, i.e. as GFR declines. The predominant cause of anemia in CKD is related to the impaired production of erythropoietin by kidneys, essential hormone for the stimulation of red blood cell production ${ }^{1}$. However, other relevant factors, such as iron deficiency, also have a major contribution to anemia in $C K D^{5}$, and its role is frequently underdiagnosed.

In CKD, anemia has been associated with heart failure, cognitive impairment, CKD progression, and higher mortality. Additionally, it has been related to a reduction in exercise capacity and decreased quality of life $\mathrm{e}^{6-8}$.

Although maintenance of stable hemoglobin levels is an important aspect of disease management, reported treatment rates of anemia in patients with CKD are typically low ${ }^{1}$. In fact, the variability and/or 
absence of guidelines regarding management and treatment of anemia within different stages of CKD patients may be preventing them from receiving optimal treatment.

Within the above described context, this study aims, through a Delphi Panel, to assess the agreement level of anemia and iron deficiency diagnosis, management, and treatment in CKD patients, by Portuguese nephrologists.

\section{METHODS}

The purpose of the study was to assess the agreement level of Nephrology physicians regarding anemia and iron deficiency diagnosis, management, and treatment, in CKD patients.

A group of seven medical experts in Nephrology and Immunohemotherapy (Transfusion Medicine) was assembled, and a focus group was conducted, in which 28 statements, divided into three main subjects, were agreed upon: A. Anemia and Iron Deficiency Diagnosis; B. Anemia and Iron Deficiency Management; C. Anemia and Iron Deficiency Treatment (Table I).

Following statement definition, a two-round Delphi Panel was conducted. The Delphi Panel method consists in a multistage process that involves interactive rounds and aims to reach consensus on a given subject, considering experts' opinion ${ }^{9}$. It is increasingly used in different areas of healthcare research, including nephrology, in which publications have been made on subjects regarding chronic kidney disease patient care ${ }^{10-12}$.

\section{Round 1}

Portuguese Society of Nephrology associates were invited by e-mail to reply to an anonymized online questionnaire. In this questionnaire, participants were asked to categorize the 28 statements presented in a randomized order using a 4-point Likert scale: fully disagree; disagree; agree; fully agree.

\section{Table I}

Statements for the web-based consultation of the consensus process.

\section{A. Diagnosis}

A1 Anemia should be defined according to the World Health Organization (WHO) definition for the general population. [Hb $<12.0 \mathrm{~g} / \mathrm{dL}$, women; $\mathrm{Hb}<13.0 \mathrm{~g} / \mathrm{dL}$, men]

A2 In my clinical practice I consider values different from the WHO definition for the diagnosis of anemia (regardless of treatment).

A3 More than the WHO definition values, I interpret hemoglobin values according to the clinical context of the patient.

A4 In Chronic Kidney Disease (CKD), the reference values for anemia should be adapted to the context/stage of the disease.

A5 The reference values for iron deficiency should be adapted to the context/stage of CKD.

\section{B. Anemia and Iron Deficiency Management}

B1 When following a patient with CKD, I request analysis of iron metabolism parameters ${ }^{1}$ only in the presence of anemia.

B2 When following a patient with CKD, I request analysis of iron metabolism parameters for patients with heart failure with reduced ejection fraction, regardless of having anemia.

B3 I request analysis of iron metabolism parameters for all dialysis patients.

B4 I request analysis of iron metabolism parameters for all kidney transplant patients.

B5 According to my clinical practice, iron metabolism parameters analysis is requested at least once a year for patients with CKD.

B6 I am well aware of the costs associated with the analysis of iron metabolism main parameters.

B7 My request for iron metabolism parameters analysis and its frequency is influenced by the associated costs.

B8 I consider reticulocyte parameters as fundamental when assessing anemia in CKD.

B9 A patient with iron deficiency without anemia does not often develop anemia.

\begin{tabular}{l|l}
\hline C1 & I start treatment in patients with hemoglobin below $10 \mathrm{~g} / \mathrm{dl}$. \\
C2 & I start treatment in patients with hemoglobin below $11 \mathrm{~g} / \mathrm{dl}$. \\
C3 & I start treatment in patients with transferrin saturation below $20 \%$. \\
C4 & I start treatment in patients with transferrin saturation below $30 \%$. \\
C5 & I consider the ferritin level for therapeutic decision in a patient with anemia and CKD. \\
C6 & In a patient with iron deficiency without anemia, I start treatment by prescribing iron supplementation. \\
C7 & The first step for treating iron deficiency anemia is to start oral iron supplementation. \\
C8 & In a kidney transplant candidate, I avoid transfusions. \\
C9 & I transfuse all patients with hemoglobin below $7 \mathrm{~g} / \mathrm{dl}$, regardless of the clinical situation. \\
C10 & I transfuse all patients with hemoglobin below $8 \mathrm{~g} / \mathrm{dl}$, regardless of the clinical situation. \\
C11 & I transfuse all patients with hemoglobin below $9 \mathrm{~g} / \mathrm{dl}$, regardless of the clinical situation. \\
C12 & A transfusion does not depend on the value of hemoglobin, but on the clinical situation of the patient. \\
C13 & Transfusing erythrocyte concentrate reduces the need for additional iron administration. \\
C14 & Blood transfusion is an iron contribution to erythropoiesis.
\end{tabular}

${ }^{1}$ Serum iron concentration, serum ferritin, and transferrin saturation. 
The target rate of response was set at 50 respondents for Round 1 and consensus agreement level was set at 70\%: all statements with at least $70 \%$ of responses "fully agree" or "fully disagree" were categorized as consensual. The statements which had not reached the consensus agreement level were then selected to be used in a second round.

\section{Round 2}

Portuguese Society of Nephrology associates who had answered in the first round were asked by e-mail to reply to an anonymized online questionnaire and categorize all the remaining statements, using the same 4-point Likert scale: fully disagree; disagree; agree; fully agree.
The target rate of response for Round 2 was set at 20 respondents and consensus agreement level was set at $70 \%$ : all the statements with at least $70 \%$ of responses "fully agree" or "fully disagree" were categorized as consensual. When statements reached combined levels of at least $70 \%$ in terms of agreement (i.e. "agree" and "fully agree") or disagreement (i.e. "disagree" and "fully disagree"), they were categorized as qualified majority.

\section{RESULTS}

\section{Round 1}

The target rate of response (number of respondents 50 ) for the first round was clearly achieved, with consistent answers from 76 participants collected.

\section{Table II}

Answers to the web-based consultation of the consensus process - Round 2.

\begin{tabular}{|c|c|c|c|c|c|c|}
\hline & Statement & $\begin{array}{l}\text { Qualified } \\
\text { Majority }\end{array}$ & \begin{tabular}{|c|} 
Fully \\
Disagree \\
$(\%)$
\end{tabular} & $\begin{array}{c}\text { Disagree } \\
(\%)\end{array}$ & $\begin{array}{c}\text { Agree } \\
(\%)\end{array}$ & $\begin{array}{c}\text { Fully } \\
\text { Agree }(\%)\end{array}$ \\
\hline A1 & $\begin{array}{l}\text { Anemia should be defined according to the World Health Organization (WHO) definition for the general population. [Hb } \\
<12.0 \mathrm{~g} / \mathrm{dL} \text {, women; } \mathrm{Hb}<13.0 \mathrm{~g} / \mathrm{dL} \text {, men] }\end{array}$ & No & 12 & 29 & 41 & 18 \\
\hline A2 & $\begin{array}{l}\text { In my clinical practice I consider values different from the WHO definition for the diagnosis of anemia (regardless of } \\
\text { treatment). }\end{array}$ & Yes & 9 & 12 & 53 & 26 \\
\hline A3 & More than the WHO definition values, I interpret hemoglobin values according to the clinical context of the patient. & Yes & 0 & 3 & 56 & 41 \\
\hline A4 & $\begin{array}{l}\text { In Chronic Kidney Disease (CKD), the reference values for anemia should be adapted to the context/stage of the } \\
\text { disease. }\end{array}$ & Yes & 0 & 9 & 56 & 35 \\
\hline A5 & The reference values for iron deficiency should be adapted to the context/stage of CKD. & Yes & 6 & 15 & 70 & 9 \\
\hline B1 & When following a patient with CKD, I request analysis of iron metabolism parameters1 only in the presence of anemia. & No & 27 & 35 & 35 & 3 \\
\hline B2 & $\begin{array}{l}\text { When following a patient with CKD, I request analysis of iron metabolism parameters for patients with heart failure with } \\
\text { reduced ejection fraction, regardless of having anemia. }\end{array}$ & No & 3 & 44 & 44 & 9 \\
\hline B3 & I request analysis of iron metabolism parameters for all dialysis patients. & Yes & 0 & 0 & 47 & 53 \\
\hline B4 & I request analysis of iron metabolism parameters for all kidney transplant patients. & Yes & 0 & 12 & 71 & 17 \\
\hline B5 & $\begin{array}{l}\text { According to my clinical practice, iron metabolism parameters analysis is requested at least once a year for patients } \\
\text { with CKD. }\end{array}$ & Yes & 6 & 15 & 53 & 26 \\
\hline B6 & I am well aware of the costs associated with the analysis of iron metabolism main parameters. & No & 3 & 32 & 47 & 18 \\
\hline B7 & My request for iron metabolism parameters analysis and its frequency is influenced by the associated costs. & No & 6 & 56 & 38 & 0 \\
\hline B8 & I consider reticulocyte parameters as fundamental when assessing anemia in CKD. & No & 3 & 65 & 29 & 3 \\
\hline B9 & A patient with iron deficiency without anemia does not often develop anemia. & No & 9 & 50 & 38 & 3 \\
\hline C1 & I start treatment in patients with hemoglobin below $10 \mathrm{~g} / \mathrm{dl}$. & Yes & 3 & 12 & 50 & 35 \\
\hline C2 & I start treatment in patients with hemoglobin below $11 \mathrm{~g} / \mathrm{dll}$. & Yes & 12 & 65 & 20 & 3 \\
\hline C3 & I start treatment in patients with transferrin saturation below $20 \%$. & Yes & 0 & 18 & 62 & 20 \\
\hline C4 & I start treatment in patients with transferrin saturation below $30 \%$. & No & 3 & 32 & 47 & 18 \\
\hline C5 & I consider the ferritin level for therapeutic decision in a patient with anemia and CKD. & Yes & 0 & 18 & 76 & 6 \\
\hline c6 & In a patient with iron deficiency without anemia, I start treatment by prescribing iron supplementation. & No & 0 & 32 & 53 & 15 \\
\hline C7 & The first step for treating iron deficiency anemia is to start oral iron supplementation. & No & 0 & 35 & 62 & 3 \\
\hline C8 & In a kidney transplant candidate, I avoid transfusions. & Yes & 0 & 0 & 35 & 65 \\
\hline c9 & I transfuse all patients with hemoglobin below $7 \mathrm{~g} / \mathrm{dl}$, regardless of the clinical situation. & No & 9 & 47 & 41 & 3 \\
\hline C10 & I transfuse all patients with hemoglobin below $8 \mathrm{~g} / \mathrm{dl}$, regardless of the clinical situation. & Yes & 53 & 47 & 0 & 0 \\
\hline C12 & A transfusion does not depend on the value of hemoglobin, but on the clinical situation of the patient. & Yes & 0 & 6 & 76 & 18 \\
\hline C13 & Transfusing erythrocyte concentrate reduces the need for additional iron administration. & No & 6 & 44 & 47 & 3 \\
\hline C14 & Blood transfusion is an iron contribution to erythropoiesis. & No & 9 & 26 & 62 & 3 \\
\hline
\end{tabular}

${ }^{1}$ Serum iron concentration, serum ferritin, and transferrin saturation. 
Consensus level was obtained for 1 out of the 28 statements. $75 \%$ $(n=57)$ of the respondents fully disagreed with [C11] 'I transfuse all patients with hemoglobin below $9 \mathrm{~g} / \mathrm{dl}$, regardless of the clinical situation'. The remaining $25 \%$ respondents $(n=19)$ disagreed with this statement.

The remaining 27 statements which had not reached the consensus agreement level on Round 1 were then used in a second, narrower, consultation.

\section{Round 2}

The target rate of response (number of respondents 20) was achieved, with consistent answers from 34 out of the 76 participants collected in the second round of the consensus process.

Consensus level was not obtained for any of the remaining 27 statements.

However, taking into account the combined levels of agreement (i.e. "agree" and "fully agree") or disagreement (i.e. "disagree" and "fully disagree"), 14 statements were categorized as qualified majority.

\section{The obtained results are summarized in Table II.}

All (100\%) specialists agreed or fully agreed with [B3] 'I request analysis of iron metabolism parameters for all dialysis patients'; and [C8] 'In a kidney transplant candidate, I avoid transfusions'. Similarly, all (100\%) physicians disagreed or fully disagreed with [C10] 'I transfuse all patients with hemoglobin below $8 \mathrm{~g} / \mathrm{dl}$, regardless of the clinical situation'.

More than $90 \%$ of the participants agreed or fully agreed with [A3] 'More than the WHO definition values, I interpret hemoglobin values according to the clinical context of the patient' (97\%); [A4] 'In Chronic Kidney Disease (CKD), the reference values for anemia should be adapted to the context/stage of the disease' (91\%); and [C12] 'A transfusion does not depend on the value of hemoglobin, but on the clinical situation of the patient' $(94 \%)$.

Additionally, more than $80 \%$ of the specialists agreed or fully agreed with [B4] 'I request analysis of iron metabolism parameters for all kidney transplant patients' (88\%); [C1] 'I start treatment in patients with hemoglobin below $10 \mathrm{~g} / \mathrm{dl}^{\prime}$ (85\%); [C3] 'I start treatment in patients with transferrin saturation below 20\%' (82\%); and [C5] 'I consider the ferritin level for therapeutic decision in a patient with anemia and CKD' (82\%).

Finally, 79\% of the respondents agreed or fully agreed with [A2] 'In my clinical practice I consider values different from the WHO definition for the diagnosis of anemia (regardless of treatment)'; [A5] 'The reference values for iron deficiency should be adapted to the context/stage of CKD'; and [B5] 'According to my clinical practice, iron metabolism parameters analysis is requested at least once a year for patients with CKD'. Similarly, $77 \%$ of the specialists disagreed or fully disagreed with [C2] 'I start treatment in patients with hemoglobin below $11 \mathrm{~g} / \mathrm{dl}^{\prime}$.

\section{DISCUSSION}

The aim of this study was to assess the agreement level on recommendations regarding anemia and iron deficiency diagnosis, management, and treatment in Chronic Kidney Disease patients. To address this goal, a Delphi Panel with Portuguese specialists in Nephrology was conducted.

Consensus was reached for just one out of the 28 statements in the panel. Additionally, despite qualified majority being achieved for 14 statements (4 on diagnosis, 3 on disease management, and 7 on treatment), this accounted for merely half of the remaining statements assessed. Overall, the results obtained clearly illustrate the heterogeneity of clinical practices in the national context, which emphasizes the need to explore this subject and develop 'real' recommendations that can be widely accepted.

In terms of Anemia and Iron Deficiency Diagnosis (Topic A), we highlight the fact that the members of the panel do not seem to consider the World Health Organization's proposed definition of anemia $^{13}$ as a suitable definition in the context of CKD. Along with the results from hemograms and iron metabolism analysis, the clinical context of the patient and stage of disease appear to be considered for the diagnosis of anemia in CKD patients (noticed by the combined levels of agreement of $97 \%$ and $91 \%$ in [A3] and [A4], respectively). This may reflect the need of some degree of flexibility in the interpretation and diagnostic value of certain clinical settings and/or laboratorial parameters. In fact, the patient's clinical situation, disease stage, and comorbidities have been previously described as factors with an impact on hemoglobin values and recommended treatment ${ }^{14,15}$.

Regarding Anemia and Iron Deficiency Management (Topic B), qualified majority was achieved regarding the request of analysis of iron metabolism parameters for all dialysis patients [B3] and for all kidney transplant patients [B4]. The European Renal Best Practice (ERBP) guidelines indicate that iron losses are greater in hemodialysis patients than in any other group ${ }^{15}$, and kidney transplant patients are known for their ongoing iron deficiency potential to delay recovery of anemia, following transplant ${ }^{16}$, which is in line with what the panelists highly agreed upon.

On the subject of Anemia and Iron Deficiency Treatment, qualified majority was achieved on statements regarding hemoglobin threshold value for starting treatment of anemia in CKD. Experts agreed with initiating treatment for hemoglobin levels inferior to $10 \mathrm{~g} / \mathrm{dl}$ [C1], and disagreed with the threshold of $11 \mathrm{~g} / \mathrm{dl}$ [C2], which is somewhat in line with the literature. Even though it was previously recommended to maintain a target hemoglobin concentration superior to $11 \mathrm{~g} / \mathrm{dl}$ in chronic kidney disease patients, there is also evidence stating that 10 $\mathrm{g} / \mathrm{dl}$ should be considered as threshold and that the optimum hemoglobin level above this concentration is uncertain ${ }^{17}$. Additionally, international guidelines mention that one should take into account the individual characteristics of the patient, as gender, age, ethnicity, and comorbidities, when setting up a target value ${ }^{17}$.

Concerning initiation of iron therapy, combined levels of agreement of $82 \%$ were achieved for starting treatment in patients with transferrin 
saturation below 20\% [C3], which is in agreement with the target defined by the ERBP guidelines ${ }^{17}$. However, there should be noted that recommendations are for patients undergoing treatment with an erythropoiesis stimulating agent, which was not prespecified in this study.

Still on the topic of treatment, concerning the hemoglobin threshold value for blood transfusion, different opinions emerged on transfusing patients with hemoglobin below $7 \mathrm{~g} / \mathrm{dl}$ [C9], $8 \mathrm{~g} / \mathrm{dl}$ [C10] or $9 \mathrm{~g} / \mathrm{dl}$ [C11], regardless of patient's clinical situation. The latter was the only statement of the Delphi Panel achieving consensus on full disagreement. Furthermore, qualified majority was observed for the intermediate hemoglobin concentration, and no consensus was achieved with the former. Our results reflect the data available on the literature, as there are no defined criteria for deciding when transfusion is to be performed, regardless of the clinical situation ${ }^{18}$. Additionally, physicians are recommended to weigh additional factors on their decision, rather than considering a hemoglobin threshold alone ${ }^{18}$ - which may, in fact, have contributed to the lack of agreement by the members of the panel. However, a clear standard of the current guidelines is that blood transfusions should be avoided in CKD patients, particularly those awaiting a kidney transplant ${ }^{18,19}$, with which the participating specialists agreed (noticed by the combined level of agreement of $100 \%$ for statement [C8]).

Regarding study limitations, along with those inherent to the Delphi technique, we believe that the number of clinicians involved in the panel was limited. Additionally, since the study was limited to the Portuguese Society of Nephrology associates, the results may be biased by their knowledge and experience on anemia in CKD. Therefore, future studies should consider an extended number of respondents and possibly additional medical specialties.

In conclusion, we can assume that there is no current consensus on diagnosis, management, and treatment of anemia in CKD patients among the nephrology community in Portugal which was included in this Delphi Panel. According to the results presented, there seems to exist a rather broad interpretation and heterogeneity of clinical practices in terms of which laboratory parameters should be assessed; which cutoff values should be used to define anemia and/or iron deficiency; and how the evaluated parameters should inform therapeutic decisions. Nonetheless, it was shown to be clear that patient's individual characteristics, clinical settings, and the physician's "clinical sense" seem to be taken into account arguably to a further extent than the currently available guidelines. It would be advantageous if future studies were to further focus on which parameters, cutoff values, and clinical settings should indeed be considered regarding anemia and iron deficiency within the context of CKD.

Disclosure of potential conflicts of interest: none declared. Support for medical writing and logistic for experts meeting was funded by Vifor Pharma Portugal. The funding source had no role in data collection and interpretation, or in writing the article.

\section{References}

1. Stauffer ME, Fan T. Prevalence of anemia in chronic kidney disease in the United States. PLoS One. 2014;9(1):2-5.

2. Webster AC, Nagler EV, Morton RL, Masson P. Chronic kidney disease. Lancet. 2017;389(10075):12381252.

3. Milman N. Anemia - Still a major health problem in many parts of the world! Ann Hematol. 2011;90(4):369-377.

4. Domenica Cappelli ni M, Motta I. Anemia in clinical practice-definition and classification: Does Hemoglobin Change With Aging? Semin Hematol. 2015;52(4):261-269.

5. Dev S, Babitt JL. Overview of iron metabolism in health and disease. Hemodial Int. 2017;21(1):S620.

6. Van Nooten FE, Green J, Brown R, Finkelstein FO, Wish J. Burden of illness for patients with nondialysis chronic kidney disease and anemia in the United States: Review of the literature. J Med Econ. 2010;13(2):241-256.

7. Mikhail A, Brown C, Williams JA, Mathrani V, Shrivastava R, Evans J, et al. Renal association clinical practice guideline on Anaemia of Chronic Kidney Disease. BMC Nephrol. 2017;18(1):1-29.

8. Drawz P, Rahman M. Chronic kidney disease. Ann Intern Med. 2015 Jun 2;162(11):ITC1-16.

9. Hasson F, Keeney S, McKenna H. Research guidelines for the Delphi survey technique. J Adv Nurs. 2000;32(4):1008-1015.

10. Bellizzi V, Bianchi S, Bolasco P, Brunori G, Cupisti A, Gambaro G, et al. A Delphi consensus panel on nutritional therapy in chronic kidney disease. J Nephrol. 2016;29(5):593-602.

11. Tu K, Bevan L, Hunter K, Rogers J, Young J, Nesrallah G. Quality indicators for the detection and management of chronic kidney disease in primary care in Canada derived from a modified Delphi panel approach. C Open. 2017;5(1):E74-E81.

12. Fukuma S, Shimizu S, Niihata K, Sada K ei, Yanagita M, Hatta T, et al. Development of quality indicators for care of chronic kidney disease in the primary care setting using electronic health data: a RAND-modified Delphi method. Clin Exp Nephrol. 2017;21(2):247-256.

13. WHO. Haemoglobin concentrations for the diagnosis of anaemia and assessment of severity. Geneva, World Heal Organ. 2011;1-6.

14. Atkinson MA, Warady BA. Anemia in chronic kidney disease. 2017;(Cd).

15. ERA-EDTA. Section I. Anaemia evalulation. Nephrol Dial Transplant. 2004;19(2):ii2-ii5.

16. Truax C, Lee S, Kenyon N, Gillespie M, Smith L, Corbett J, et al. ncidence of iron deficiency in kidney transplant candidates based on the updated KDIGO Guideline for Anemia, the [abstract]. Am J Transpl. 2013;13(suppl 5).

17. ERA-EDTA. Section II. Targets for anaemia treatment. Nephrol Dial Transplant. 2004;19(2):ii6-ii15.

18. Kidney Disease: Improving Global Outcomes (KDIGO) Anemia Work Group. KDIGO Clinical Practice Guideline for Anemia in Chronic Kidney Disease. Kidney Int Suppl. 2012;2(4):279-335.

19. ERA-EDTA. Section III. Treatment of renal anaemia. Nephrol Dial Transplant. 2004;19(2):16-31.

\section{Correspondence to:}

Ana Macedo

Alameda Fernão Lopes 16A, 7ํAndar, Escritório 1, 1495-190 Algés, Portugal E-mail: amacedo@keypoint.pt

Mobile: +351 214096079 